\title{
Consistency of Primordial Black Hole Dark Matter with LIGO/Virgo Merger Rates
}

\author{
Karsten Jedamzik \\ Laboratoire de Univers et Particules de Montpellier, UMR5299-CNRS, Universite de Montpellier, \\ 34095 Montpellier, France
}

(Received 14 July 2020; revised 30 October 2020; accepted 12 January 2021; published 4 February 2021)

\begin{abstract}
The LIGO/Virgo Collaboration has by now observed or constrained the gravitational merger rates of different classes of compact objects. We consider the possibility that the bulk of these mergers are primordial black hole $(\mathrm{PBH})$ mergers of $\mathrm{PBHs}$ formed during the QCD epoch making up the entirety of the dark matter. Having shown in a companion paper that mergers due to the initial binary population formed in the early Universe are likely negligible, we compute current merger rates in $\mathrm{PBH}$ clusters in which the typical PBH resides. We consider two scenarios: (i) the PBH mass function dictated by the QCD equation of state and (ii) the $\mathrm{PBH}$ mass function dictated by the existence of a peak in the inflationary perturbation spectrum. In the first scenario, which is essentially parameter-free, we reproduce very well the merger rates for heavy BHs, the merger rate of mass-asymmetric BHs such as GW190814, a recently discovered merger of a $23 M_{\odot}$ black hole with a $2.6 M_{\odot}$ object, and can naturally explain why LIGO/Virgo has not yet observed mergers of two light PBHs from the dominant $\sim 1 M_{\odot} \mathrm{PBH}$ population. In the second scenario, which has some parameter freedom, we match well the observed rate of heavy PBHs but can currently not explain the rate for mass-asymmetric events. In either case, it is tantalizing that in both scenarios PBH merger rates made with a minimum of assumptions match most LIGO/Virgo observed rates very well.
\end{abstract}

DOI: 10.1103/PhysRevLett.126.051302

With the technological advancement of the LIGO and Virgo gravitational wave detectors, a new field of observational astrophysics and cosmology has emerged. LIGO/ Virgo by now routinely observe the gravitational wave emission of mergers of black holes $(\mathrm{BH})$ or neutron star (NS) binaries out to very large cosmic distances. Each observational run seems to provide a new surprise: LIGO O1 the detection of a very massive binary of two $\sim 30 M_{\odot}$ $\mathrm{BHs}$ [1], O2 the detection of many such massive binaries with often close to equal masses of the binary components and very low spin $[2,3]$, and $\mathrm{O} 3$ the detection of a binary merger, with one of the binaries seemingly being too massive for a NS and too light for being a BH [4]. These observations have confronted the theoretical community of stellar population models with new challenges. It seems not impossible that $\sim 30 M_{\odot}$ may be produced as a result of star formation (and evolution) if it happens in a low-metallicity environment $[5,6]$ or $\sim 2.6 M_{\odot}$ BHs are made by the merger of two lighter NSs as in the case of GW170817 (see [7,8] for alternative explanations).

In this Letter, we follow an alternative path, investigating if the observed objects could be primordial black holes (PBHs) formed during the QCD epoch (for general reviews on PBHs, cf. $[9,10])$. It has been known for some while that equation of state (EOS, hereafter) effects during the QCD epoch lead to an enhanced PBH formation on a particular scale [11-13], provided the underlying inflationary density fluctuations are approximately scale invariant. Recent investigations of the resulting mass spectrum [14-16], under the assumption of Gaussianity for the fluctuations, and taking into account the accurate zero chemical potential EOS [17,18], reveal a well-defined peak on a scale somewhere around $0.5-2 M_{\odot}$ containing most of the PBHs and a shoulder between $\sim 8$ and $50 M_{\odot}$ containing a smaller mass fraction $f_{M} \approx 10^{-2}$ of PBHs. Here the uncertainties are mostly due to the semianalytic nature of the estimates and may well be addressed by complete general-relativistic numerical simulations of the PBH formation process [19-21]. An alternative scenario of $\mathrm{PBH}$ formation during the QCD epoch may be imagined if inflation accidentally has left a peak in the power spectrum on the required scale, for example, due to a temporary flattening of the inflaton potential [22,23] (see [24] for another alternative). Many inflationary scenarios leading to PBH formation have been investigated, too numerous to cite here. In what follows, we will investigate these two alternative explanations for the LIGO/Virgo observations. Our complete attention is on the prediction of binary merger rates. Throughout this Letter, we will assume that PBHs contribute the entirety of the cosmic dark matter.

It had been believed for some time now that $\sim 1-30 M_{\odot}$ PBHs cannot contribute the entirety of the dark matter, rather only a very small fraction, as it was argued that very eccentric and hard binaries forming shortly before cosmological matter-radiation equality would lead to current BH-BH merger rates incompatible with those inferred by LIGO/Virgo [25-31]. In a recent paper, appearing two days before the announcement of GW190814, we have shown 
that the conclusions about the viability of PBH dark matter are drastically modified when considering three-body interactions between the two binary members and a third bypassing PBH in early PBH clusters [32]. In fact, we have found for $M=1 M_{\odot}$ that the formerly predicted approximate merger rate $\mathcal{M}_{M_{\odot}} \approx 1.25 \times 10^{6} \mathrm{Gpc}^{-3} \mathrm{yr}^{-1}$ is reduced to $\mathcal{M} \approx 40 \mathrm{Gpc}^{-3} \mathrm{yr}^{-1}$ by three-body interactions. The rate is likely even lower if more frequent encounters are taken into account. This rate should be compared to the current upper limit $\mathcal{M}_{M_{\odot}} \approx 5.2 \times 10^{3} \mathrm{Gpc}^{-3} \mathrm{yr}^{-1}$ from LIGO/Virgo. It is important to note that the prediction of these rates is subject to one important uncertainty, i.e., the fraction of binaries which never entered clusters, $f_{\text {free }}$. We will return to this point below.

Building on prior results [29,33-35] (cf. also to [36]), we have argued in Ref. [32] that PBHs form clusters at very high redshifts which evaporate later due to the continuous loss of PBHs in the high-energy tail of the MaxwellBoltzmann energy distribution. We have found that clusters of $N_{\mathrm{cl}} \approx 1300$ with approximate densities $n_{\mathrm{cl}} \approx 3.6 / \mathrm{pc}^{3}$ having formed at redshifts $z \approx 85$ are evaporating at the current epoch. We will use these values as reference values for the typical environment in which $\mathrm{PBHs}$ are at the present epoch. However, all given merger rates will include the full dependence on clusters properties, given by the PBH number in a cluster $N_{\mathrm{cl}}$ and the PBH masses.

$P B H$ formation during the QCD epoch with approximately scale-invariant inflationary perturbations.-We stress here that when referring to scale-invariant fluctuations we do not imply that these fluctuations have the same amplitude as on the much larger cosmic microwave background scales but rather are approximately scale invariant over the 2-3 $e$-folds during inflation which produce perturbations on the QCD scales considered here. This could be accomplished by a broad peak produced during inflation. Alternatively, in the case of a power-law spectrum, one can show that, in order to not overproduce smaller PBHs above the Hawking mass, the spectral index has to be redder than $n_{s} \approx 0.973$. In addition, the spectrum has to have a significant—orders of magnitude-decrease on large scales. We will approximate the mass function of such PBHs by a simple bimodal distribution with a peak mass scale $M_{s}$ containing the majority of PBHs, i.e., $f_{M}\left(M_{s}\right) \approx 1$, and a larger mass scale $M_{b} \gg M_{s}$ containing a much smaller mass fraction $f_{M}\left(M_{b}\right) \ll 1$. Given the simplicity of our estimate, any more detailed treatment is currently not warranted. We now proceed to the calculation of merger rates. We will assume that, as has been explicitly shown in the case of $M_{s}-M_{s} \mathrm{PBH}$ binaries, with $M_{s}=M_{\odot}$, three-body $\mathrm{PBH}$ interactions will significantly modify the initial eccentricity $e$ and semimajor axis $a$ distributions $P(a, e)$ for $M_{s}-M_{b}$ and $M_{b}-M_{b}$ binaries, such that their rates are well below the current inferred rates for such mergers. These assumptions seem very reasonable also, since for typical $f_{M}\left(M_{b}\right) \approx 10^{-2}$ there should be many fewer $M_{b}-M_{b}$ binaries than when $f_{M}\left(M_{b}\right) \approx 1$. Similar arguments apply to the frequency of $M_{s}-M_{b}$ binaries. With the initial binary population disrupted and the subsequent formation of very hard and eccentric binaries improbable, the current merger rate would be dominated by direct capture of two single PBHs.

Single PBHs may coalesce by emission of gravitational radiation during close encounters. The fraction of PBHs which merge in this way over a time interval $\Delta t$ is given by

$$
f_{\text {direct }}=\frac{\Delta n_{\mathrm{PBH}}}{n_{\mathrm{PBH}}}=\frac{1}{2} \sigma_{\text {capt }} v n \Delta t,
$$

where

$$
\begin{aligned}
\sigma_{\text {capt }}= & 2 \pi\left(\frac{85 \pi}{6 \sqrt{2}}\right)^{2 / 7}\left(\frac{G}{c}\right)^{2}\left(M_{1}+M_{2}\right)^{10 / 7} \\
& \times\left(M_{1} M_{2}\right)^{2 / 7}\left(\frac{v}{c}\right)^{-18 / 7}
\end{aligned}
$$

is the capture cross section [37]. Here $G$ is the gravitational constant, $c$ is the speed of light, $M_{1}$ and $M_{2}$ are the two BH masses, $v$ is their relative velocity, and $n$ is the number density of the BHs. Such direct mergers had been considered in Refs. [38,39] though not within clusters formed due to Poissonian fluctuations. They were later on deemed subdominant due to the initial binary population. The direct capture rate today may be estimated via

$$
\mathcal{M}=\left(\frac{f_{\text {direct }}}{\Delta t}\right)\left(\frac{f_{M}\left(M_{\mathrm{PBH}}\right) \rho_{\mathrm{PBH}}^{\mathrm{avg}}}{M_{\mathrm{PBH}}}\right),
$$

where $\rho_{\mathrm{PBH}}^{\mathrm{avg}}$ is the present cosmic average $\mathrm{PBH}$ mass density. Using the above equations on the merger rate of two light PBHs, we find

$\mathcal{M}_{M_{s}-M_{s}}^{d} \approx 639 \frac{1}{\mathrm{Gpc}^{3} \mathrm{yr}}\left(\frac{M_{s}}{1 M_{\odot}}\right)^{-11 / 21}\left(\frac{N_{\mathrm{cl}}}{1300}\right)^{-137 / 84}$

well below the current observational limit [40].

After formation, clusters attain virial equilibrium quite rapidly. In virial equilibrium, the typical kinetic energies $E_{k}$ of heavy and light PBHs are the same, such that $v\left(M_{b}\right) \equiv v_{M_{b}} \simeq v_{\mathrm{cl}} \sqrt{M_{s} / M_{b}}$, where $v_{\mathrm{cl}}=v\left(M_{s}\right)$. This leads to the sinking of heavy PBHs to the center of the cluster. In this Letter, we will adopt a simple top hat (i.e., constant density) model for the clusters. More realistically, one should probably choose a Plummer or King model as often used for globular clusters; however, for this simple first-order estimate, a top hat model may be an acceptable approximation. In the top hat model, the gravitational potential at radii $r<r_{\mathrm{cl}}$ may be approximated by $V_{p} \simeq-\left(G M_{\mathrm{cl}} M_{b} / r_{\mathrm{cl}}\right)\left(r / r_{\mathrm{cl}}\right)^{2}$, where $M_{\mathrm{cl}}$ and $r_{\mathrm{cl}}$ are the total cluster mass and cluster virial radius, respectively. 
Note that, strictly speaking, we would need to include selfconsistently the gravitational potential at the center of the cluster provided by the heavy PBHs. However, since $f_{M}\left(M_{b}\right)$ is assumed to be small, this effect is only of the order of unity and will here be omitted. Assuming virial equilibrium for the heavy $M_{b}$ PBHs as well, i.e., $E_{k} \simeq-V_{p} / 2$, we may compute the virial radius for the heavy $\mathrm{PBHs}$ to be $r_{M_{b}}^{\mathrm{vir}} \simeq r_{\mathrm{cl}}\left(v_{M_{b}} / v_{M_{s}}\right) \simeq r_{\mathrm{cl}} \sqrt{M_{s} / M_{b}}$. That implies their number density is increased by a factor of ${\sqrt{M_{b} / M_{s}}}^{3}$. Given this, one may compute the ratio of heavy to light PBH number densities at the center of the cluster to be $f_{M}\left(M_{b}\right) \sqrt{M_{b} / M_{s}}$, which is much larger then the cosmic average of $f_{M}\left(M_{b}\right)\left(M_{s} / M_{b}\right)$. This estimate of the number density of the heavy PBHs, together with the direct merger cross section, may be utilized to compute the present-day merger rate of two large PBHs with mass $M_{b}$. In particular, one may employ Eqs. (1)-(3) with the values of $v=v_{M b} \simeq v_{\mathrm{cl}} \sqrt{M_{s} / M_{b}}$ and $n=n_{\mathrm{cl}} f_{M}\left(M_{b}\right){\sqrt{M_{b} / M_{s}}}^{3}$. This yields

$$
\begin{aligned}
\mathcal{M}_{M_{b}-M_{b}}^{d} \approx & 152 \frac{1}{\mathrm{Gpc}^{3} \mathrm{yr}}\left(\frac{f_{M}\left(M_{b}\right)}{10^{-2}}\right)^{2}\left(\frac{M_{b}}{30 M_{\odot}}\right)^{16 / 7} \\
& \times\left(\frac{M_{s}}{1 M_{\odot}}\right)^{-59 / 21}\left(\frac{N_{\mathrm{cl}}}{1300}\right)^{-137 / 84}
\end{aligned}
$$

coming very close to that observed. However, it is important to note that a typical cluster of $N_{\mathrm{cl}}=1300$ on average does not even include a single heavy $\mathrm{PBH}$, due to the rarity of massive PBHs. Requiring that the average number of massive PBHs in a cluster is two, i.e., $\left(M_{s} / M_{b}\right) f_{M}\left(M_{b}\right) N_{\mathrm{cl}}=2$, a cluster has to be of size $N_{\mathrm{cl}}=6000$ for an example $M_{b}=30 M_{\odot}$, implying a rate of $\mathcal{M}_{30 M_{\odot}-30 M_{\odot}}^{d} \sim 12 \mathrm{Gpc}^{-3} \mathrm{yr}^{-1}$. These values bracket very well those inferred from the LIGO/Virgo Collaboration.

Next, we calculate the merger rate of light PBHs with heavy PBHs. Here the appropriate values to enter in Eq. (1) are $v=v_{\mathrm{cl}}$, since $v_{\mathrm{cl}} \gg v_{M b}$ and $n=n_{\mathrm{cl}}$. This yields

$$
\begin{aligned}
\mathcal{M}_{M_{b}-M_{s}}^{d} \approx & 28.2 \frac{1}{\mathrm{Gpc}^{3} \mathrm{yr}}\left(\frac{f_{M}\left(M_{b}\right)}{10^{-2}}\right)\left(\frac{M_{b}}{30 M_{\odot}}\right)^{5 / 7} \\
& \times\left(\frac{M_{s}}{1 M_{\odot}}\right)^{-26 / 21}\left(\frac{N_{\mathrm{cl}}}{1300}\right)^{-137 / 84} .
\end{aligned}
$$

Again requiring that on average there is at least one heavy $\mathrm{PBH}$ in the cluster, we find, for $M_{s}=1 M_{\odot}$ and $M_{b}=30 M_{\odot}$ as an example $N_{\mathrm{cl}}=3000$, yielding a rate $\mathcal{M}_{30 M_{\odot}-1 M_{\odot}}^{d} \sim 7.1 \mathrm{Gpc}^{-3} \mathrm{yr}^{-1}$. This should be compared to the by LIGO/Virgo estimated rate $\mathcal{M}_{30}^{d} M_{\odot}-1 M_{\odot} \sim$ $1-23 \mathrm{Gpc}^{-3} \mathrm{yr}^{-1}$ for GW190814-like events. It is tantalizing that both merger rates in this simple estimate come so close to the observed values (see [41] for the merger rate if only one object is a PBH).
$P B H$ formation during the $Q C D$ epoch with an inflationary peak.-We now consider scenarios where the preexisting inflationary fluctuations necessary for $\mathrm{PBH}$ formation accidentally have a peak on the mass scale $M_{\mathrm{PBH}}=30 M_{\odot}$. We note that in contrast to the prior scenario, where there was only one free parameter, the fraction of dark matter in PBHs, which we assumed to be unity, the scenario here has a second free parameter $M_{\mathrm{PBH}}$. Simulations in Ref. [32] had been performed only for $M_{\mathrm{PBH}}=1 M_{\odot}$, so a priori we cannot be sure if similar destruction rates of the initial population of very hard and eccentric binaries apply. However, it is not too difficult to show that physics of binary destruction in clusters formed due to Poisson noise in the number of PBHs is independent of the mass scale $M_{\mathrm{PBH}}$ and thus scale invariant. This applies also to the formation and evaporation of clusters. We may therefore directly apply our findings in Ref. [32] to the current problem, implying that present-day mergers in PBH clusters due to the initial population are negligible. We may then use the above equations with $v=v_{\mathrm{cl}}$ and $n=n_{\mathrm{cl}}$ to find the following rate:

$$
\mathcal{M}_{M_{b}-M_{b}}^{d} \approx 108 \frac{1}{\mathrm{Gpc}^{3} \mathrm{yr}}\left(\frac{M_{b}}{30 M_{\odot}}\right)^{-11 / 21}\left(\frac{N_{\mathrm{cl}}}{1300}\right)^{-137 / 84} \text {. }
$$

Again, it is tantalizing that this rate is so close to that inferred by LIGO/Virgo. An estimate of the mergers of heavy $\sim 20-30 M_{\odot}$ with light $\sim 1-3 M_{\odot}$ PBHs is difficult, as there is no clear prediction, unlike in the QCD EOS scenario. However, we may speculate that there exists a broad and symmetric peak and that there is some enhancement of $\mathrm{PBH}$ formation on the $\sim M_{\odot} \mathrm{PBH}$ scale due to the QCD equation of state. Since most PBHs are heavy, they will dominate the properties of clusters. In the attempt of light $\mathrm{PBHs}$ to attain kinetic energy equilibrium in $\mathrm{PBH}$ clusters, they will quickly reach velocities much beyond the virial velocity and evaporate. One may thus imagine current galaxies and clusters of galaxy halos with a smooth light PBH background, but with heavy PBH clusters, which dominate the gravitational potential of the galaxy or cluster. We have estimated the light-heavy PBH merger rate in this context. It would be given by the probability that a light PBH enters into a PBH cluster and subsequently coalesces by a direct merger. We found the resultant merger rates orders of magnitude below those observed. Of course, it is conceivable that light and heavy PBHs contribute almost similarly to the dark matter, going again a step toward the EOS scenario. In this case, every heavy PBH cluster would be the center of a light $\mathrm{PBH}$ cluster with the latter having a much larger virial radius. Thus, merger rates could be parametrically enhanced. We leave such considerations for future study.

The typical environment PBHs exist in the current epoch.-All these merger rates are displayed in Figs. 1-3 


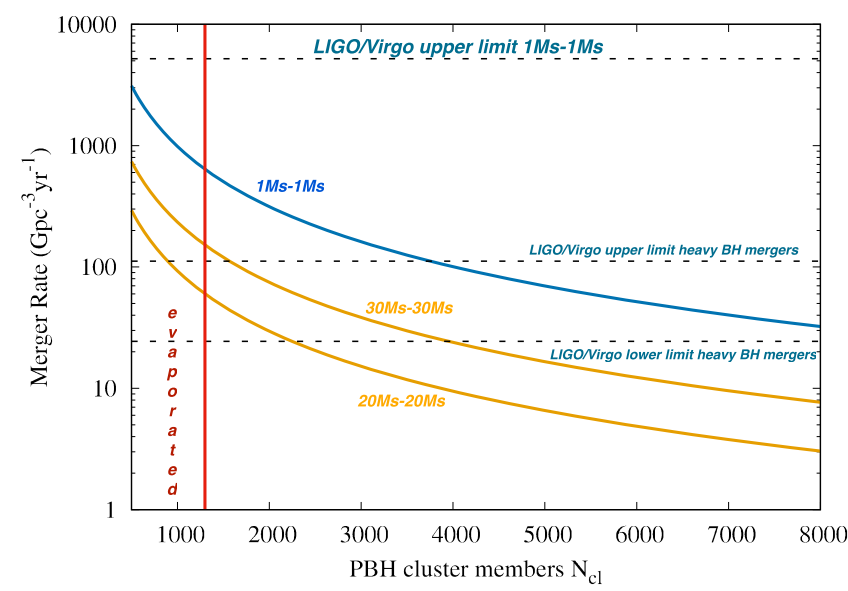

FIG. 1. The predicted current merger rates of two equal mass PBHs as a function of the number of PBH cluster members $N_{\mathrm{cl}}$ they reside in today. Results for unpeaked inflationary perturbations are shown. Small PBH clusters with $N_{\mathrm{cl}}$ left to the vertical red line have evaporated by today [45] and do not contribute. The upper horizontal dashed line shows the $90 \%$ confidence upper limit of the LIGO/Virgo rate on coalescence of two $1 M_{\odot} \mathrm{BHs}$ and should be compared to the dark blue line given by Eq. (4). The lower two dashed horizontal lines show the $90 \%$ confidence LIGO/Virgo range of the detected merger rate of heavy PBHs. These should be compared to the two orange lines [cf. Eq. (5)], showing the $\mathrm{PBH}$ merger rate range for heavy $20 M_{\odot}<M_{\mathrm{PBH}}<$ $30 M_{\odot}$ PBHs. It is important to note that most PBHs are expected to reside in $\mathrm{PBH}$ clusters just right to the red line, above the current cluster evaporation limit.

as a function of the number of cluster members $N_{\mathrm{cl}}$ they reside in. It is important to realize that, though $N_{\mathrm{cl}}$ was chosen for the $x$ axis in those figures, $N_{\mathrm{cl}}$ is highly constrained. $\mathrm{PBH}$ clusters below a critical $N_{\mathrm{cl}}^{\mathrm{cr}}$ have evaporated by now. In the spirit of hierarchical structure formation, clusters of smaller $N_{\mathrm{cl}}^{s}$ but with $N_{\mathrm{cl}}^{s}>N_{\mathrm{cl}}^{\mathrm{cr}}$ are embedded in clusters of even larger $N_{\mathrm{cl}}^{b}$. As a somewhat oversimplified example, one would expect ten clusters with $N_{\mathrm{cl}}^{s}=1300$ to make up one cluster of $N_{\mathrm{cl}}^{b}=13000$. The smallest clusters not yet evaporated are, therefore, the basic building blocks. From detailed simulations of CDM structure formation, one has the experience that tidal forces are important in destroying some substructure. However, such arguments do not play an important role for PBH clusters on small scales formed from Poissonian noise, as the power spectrum on small scales is very different than that of CDM. PBH cluster densities scale as $n_{\mathrm{cl}} \sim N_{\mathrm{cl}}^{-3 / 2}$ such that the smallest clusters are much more dense and may not tidally be destroyed. Note that the existence of a population of high redshift clusters may explain a long-standing problem in cosmology, a significant excess in the cosmic infrared background on small scales [42-44].

Mergers from PBHs which never entered clusters.-The merger rate of the initial population of hard and eccentric binaries is large $[25,46]$ :

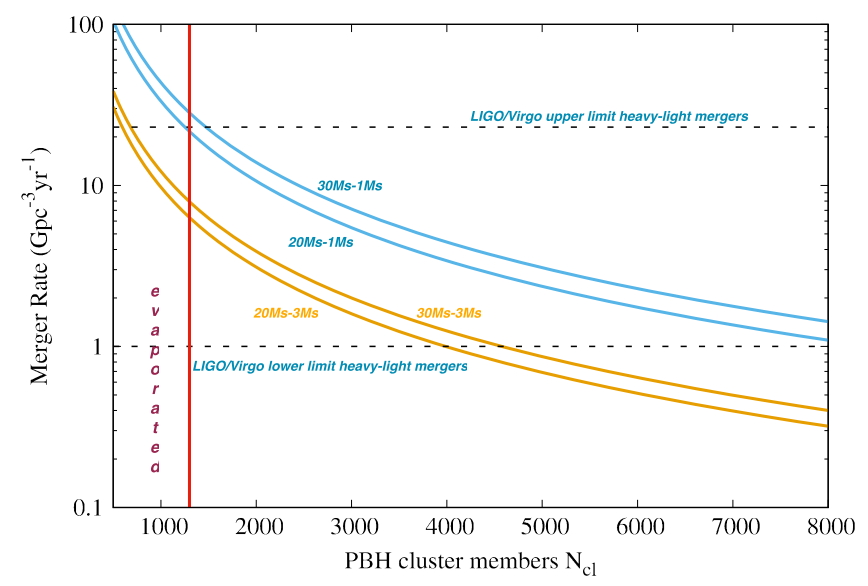

FIG. 2. Merger rates of light PBHs with heavy PBHs as predicted by Eq. (6). Results for unpeaked inflationary perturbations are shown. The two dotted lines present the by LIGO/Virgo inferred upper and lower limit on this type of event, whereas the red line shows the critical $N_{\mathrm{cl}}^{\mathrm{cr}}$ of clusters which have evaporated today. Four binary mass combinations are considered: $\left(M_{1} / M_{\odot}, M_{2} / M_{\odot}\right)=(30,1),(30,3),(20,1)$, and $(20,3)$, where the blue lines are for $M_{2}=1 M_{\odot}$ and the orange lines are for $M_{2}=3 M_{\odot}$ PBHs.

$\mathcal{M}^{i b} \approx 1.25 \times 10^{6} f_{\text {free }} f_{M}\left(M_{\mathrm{PBH}}\right) \frac{1}{\mathrm{Gpc}^{3} \mathrm{yr}}\left(\frac{M_{\mathrm{PBH}}}{1 M_{\odot}}\right)^{-32 / 37}$,

implying that the fraction of PBHs which never entered a cluster has to be smaller than

$$
\begin{array}{ll}
f_{\text {free }} f_{M}\left(M_{\mathrm{PBH}}\right) \lesssim 4.2 \times 10^{-3} & \text { for } M_{\mathrm{PBH}}=1 M_{\odot}, \\
f_{\text {free }} f_{M}\left(M_{\mathrm{PBH}}\right) \lesssim 1.7 \times 10^{-3} & \text { for } M_{\mathrm{PBH}}=30 M_{\odot}
\end{array}
$$

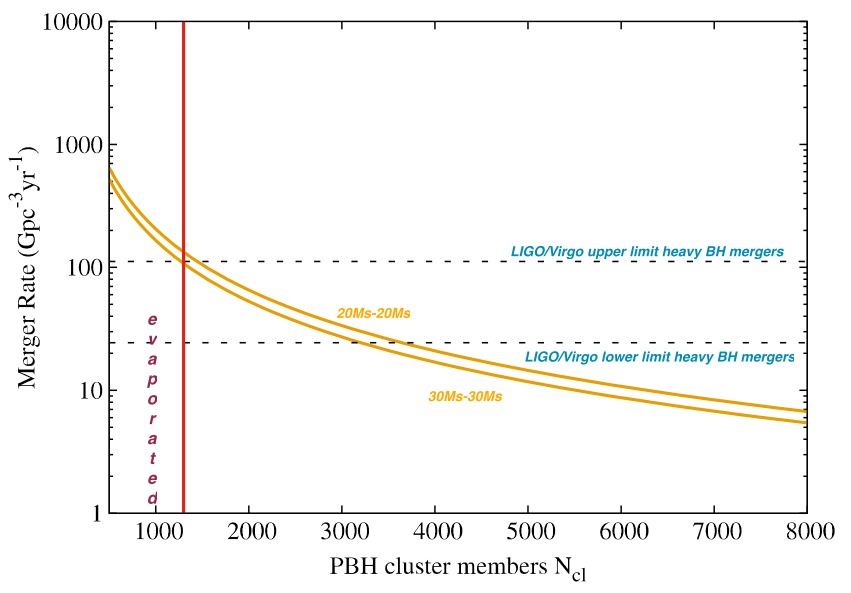

FIG. 3. Merger rates for inflationary perturbations with a peak on the QCD scale as predicted by Eq. (7). Shown is the equal mass merger rate for heavy $\mathrm{PBHs}$ in the range $20 M_{\odot}<M_{\mathrm{PBH}}<30 M_{\odot}$, as in Fig. 1. 
in order to not exceed their respective limits (see [30] for a related discussion). For PBH masses somewhat smaller than a solar mass, these limits are significantly relaxed due to a sensitivity loss of LIGO/Virgo. Such small $f_{\text {free }}$ [assuming $f_{M}\left(M_{\mathrm{PBH}}\right) \approx 1$ ] are close to what one finds in CDM simulations [47]. However, values for $f_{\text {free }}$ could be even smaller in PBH dark matter scenarios due to the much earlier onset of structure formation and the initial zero velocities of PBHs. Nevertheless, it is important that $f_{\text {free }}$ is determined by dedicated PBH structure formation simulations such as the one in Ref. [35].

Taken at face value, the QCD EOS scenario for PBH dark matter seems observationally challenged due to microlensing constraints in our Galactic halo $[48,49]$ and due to high-magnification microlensing observations in giant arcs due to caustic crossings [50]. On the other hand, Ref. [48] reported a detection of a significant population of $\sim 0.5 M_{\odot}$ unidentified objects, probably in the Galactic halo. A scenario of PBHs on the $\sim 30 M_{\odot}$ scale due to an inflationary peak is less constrained by galactic microlensing but still constrained by observations of giant arcs. As none of these constraints are overly stringent, and since such analysis has to be adapted to the particularity of PBHs being in clusters, we believe microlensing constraints deserve further scrutiny.

In conclusion, we pause for a moment and have a look from a distance at our findings. Scenarios where PBHs form during the QCD epoch have essentially only one free parameter $f_{\mathrm{PBH}}$, the contribution of such $\mathrm{PBHs}$ to the cosmic dark matter. Once this parameter has been fixed, where we chose the natural Occam's razor value of $f_{\mathrm{PBH}}=1$, there remains no further parametric freedom. Everything else is simply dictated by known physics. In this highly constrained setting, PBHs formed during the QCD epoch can (predict) postdict the mass scale of $\sim 30 M_{\odot}$ for PBHs observed by LIGO/Virgo, the current merger rate of such PBHs observed by LIGO/Virgo, the current merger rate of light $\mathrm{PBHs}$ with heavy ones observed by LIGO/Virgo, and the current nonobservation of mergers on the fundamental $\sim 1 M_{\odot} \mathrm{PBH}$ scale. It may be that nature has not chosen this pathway but, if not, has confronted us with an astonishing coincidence.

I acknowledge useful exchanges with Yacine AliHamoud, Krzysztof Belczynski, Derek Inman, Alexander Kashlinsky, Levon Pogosian, Ville Vaskonen, and Hardi Veermae.

[1] B. Abbott et al. (LIGO Scientific and Virgo Collaborations), Phys. Rev. Lett. 116, 061102 (2016).

[2] B. Abbott et al. (LIGO Scientific and Virgo Collaborations), Phys. Rev. X 9, 031040 (2019).

[3] B. Abbott et al. (LIGO Scientific and Virgo Collaborations), Astrophys. J. Lett. 882, L24 (2019).
[4] R. Abbott et al. (LIGO Scientific and Virgo Collaborations), Astrophys. J. 896, L44 (2020).

[5] K. Belczynski, T. Bulik, C. L. Fryer, A. Ruiter, J. S. Vink, and J. R. Hurley, Astrophys. J. 714, 1217 (2010).

[6] K. Belczynski, M. Dominik, T. Bulik, R. O’Shaughnessy, C. Fryer, and D. E. Holz, Astrophys. J. Lett. 715, L138 (2010).

[7] T. Broadhurst, J. M. Diego, and G. F. Smoot, arXiv: 2006.13219.

[8] M. Safarzadeh and A. Loeb, Astrophys. J. Lett. 899, L15 (2020).

[9] M. Y. Khlopov, Res. Astron. Astrophys. 10, 495 (2010).

[10] B. Carr, K. Kohri, Y. Sendouda, and J. Yokoyama, arXiv: 2002.12778.

[11] K. Jedamzik, Phys. Rev. D 55, R5871 (1997).

[12] K. Jedamzik, Phys. Rep. 307, 155 (1998).

[13] K. Jedamzik and J. C. Niemeyer, Phys. Rev. D 59, 124014 (1999).

[14] C. T. Byrnes, M. Hindmarsh, S. Young, and M. R. S. Hawkins, J. Cosmol. Astropart. Phys. 08 (2018) 041.

[15] B. Carr, S. Clesse, J. Garcia-Bellido, and F. Kuhnel, Phys. Dark Universe 31, 100755 (2021).

[16] J. Sobrinho and P. Augusto, Mon. Not. R. Astron. Soc. 496, 60 (2020).

[17] S. Borsanyi et al., Nature (London) 539, 69 (2016).

[18] T. Bhattacharya, M. I. Buchoff, N. H. Christ, H.-T. Ding, R. Gupta, C. Jung, F. Karsch, Z. Lin, R. D. Mawhinney, G. McGlynn, S. Mukherjee, D. Murphy, P. Petreczky, D. Renfrew, C. Schroeder, R. A. Soltz, P. M. Vranas, and H Yin (HotQCD Collaboration), Phys. Rev. Lett. 113, 082001 (2014).

[19] J. C. Niemeyer and K. Jedamzik, Phys. Rev. Lett. 80, 5481 (1998).

[20] J. C. Niemeyer and K. Jedamzik, Phys. Rev. D 59, 124013 (1999).

[21] I. Musco, J. C. Miller, and L. Rezzolla, Classical Quantum Gravity 22, 1405 (2005).

[22] P. Ivanov, P. Naselsky, and I. Novikov, Phys. Rev. D 50, 7173 (1994).

[23] J. S. Bullock and J. R. Primack, Phys. Rev. D 55, 7423 (1997).

[24] A. Dolgov and J. Silk, Phys. Rev. D 47, 4244 (1993).

[25] M. Sasaki, T. Suyama, T. Tanaka, and S. Yokoyama, Phys. Rev. Lett. 117, 061101 (2016); 121, 059901(E) (2018).

[26] Y. Ali-Haïmoud, E. D. Kovetz, and M. Kamionkowski, Phys. Rev. D 96, 123523 (2017).

[27] G. Ballesteros, P. D. Serpico, and M. Taoso, J. Cosmol. Astropart. Phys. 10 (2018) 043.

[28] T. Bringmann, P. F. Depta, V. Domcke, and K. SchmidtHoberg, Phys. Rev. D 99, 063532 (2019).

[29] M. Raidal, C. Spethmann, V. Vaskonen, and H. Veerme, J. Cosmol. Astropart. Phys. 02 (2019) 018.

[30] V. Vaskonen and H. Veermäae, Phys. Rev. D 101, 043015 (2020).

[31] V. De Luca, G. Franciolini, P. Pani, and A. Riotto, J. Cosmol. Astropart. Phys. 06 (2020) 044.

[32] K. Jedamzik, J. Cosmol. Astropart. Phys. 09 (2020) 022.

[33] N. Afshordi, P. McDonald, and D. Spergel, Astrophys. J. Lett. 594, L71 (2003).

[34] J. R. Chisholm, Phys. Rev. D 73, 083504 (2006). 
[35] D. Inman and Y. Ali-Haïmoud, Phys. Rev. D 100, 083528 (2019).

[36] M. Trashorras, J. Garca-Bellido, and S. Nesseris, arXiv: 2006.15018.

[37] H. Mouri and Y. Taniguchi, Astrophys. J. Lett. 566, L17 (2002).

[38] S. Bird, I. Cholis, J. B. Muñoz, Y. Ali-Haïmoud, M. Kamionkowski, E. D. Kovetz, A. Raccanelli, and A. G. Riess, Phys. Rev. Lett. 116, 201301 (2016).

[39] S. Clesse and J. Garca-Bellido, Phys. Dark Universe 18, 105 (2017).

[40] B. Abbott et al. (LIGO Scientific and Virgo Collaborations), Phys. Rev. Lett. 123, 161102 (2019).

[41] K. Vattis, I. S. Goldstein, and S. M. Koushiappas, Phys. Rev. D 102, 061301 (2020).

[42] A. Kashlinsky, R. Arendt, J. C. Mather, and S. Moseley, Astrophys. J. Lett. 654, L5 (2007); 657, L131(E) (2007).
[43] A. Kashlinsky, Astrophys. J. Lett. 823, L25 (2016).

[44] A. Kashlinsky, R. G. Arendt, F. Atrio-Barandela, N. Cappelluti, A. Ferrara, and G. Hasinger, Rev. Mod. Phys. 90, 025006 (2018).

[45] J. Binney and S. Tremaine, Galactic Dynamics, 2nd ed. (Princeton University Press, Princeton, 2008).

[46] T. Nakamura, M. Sasaki, T. Tanaka, and K. S. Thorne, Astrophys. J. Lett. 487, L139 (1997).

[47] J. Stucker, P. Busch, and S. D. White, Mon. Not. R. Astron. Soc. 477, 3230 (2018).

[48] C. Alcock et al. (MACHO Collaboration), Astrophys. J. 542, 281 (2000).

[49] P. Tisserand et al. (EROS-2 Collaboration), Astron. Astrophys. 469, 387 (2007).

[50] M. Oguri, J. M. Diego, N. Kaiser, P. L. Kelly, and T. Broadhurst, Phys. Rev. D 97, 023518 (2018). 